\title{
Puzur-Mama und die Reise des Königs
}

\author{
von Konrad Volk - München
}

Dic "Reisen" sargonischer Herrscher in den mesopotamischen Süden waren stets mit enormen Leistungen und Verpflichtungen der Kommunen des Südens verbunden. In der Spätzeit des Königs Sarkališarri scheint sich diesc: Situation jedoch grundlegend zu ändern: Puzur-Muma, der Ensi von Lagaś-Girsu, fordert Territorialgarantien(?) von Sarkajiăarri, eignet aich wenig später (nach Sarkališarrīa Tod?) den Titel lugal "König“ an und betreibt in seiner einzigen erhsltenen Königsinschrift seine adärjuate Selbstdarstellung.

Historische Inschriften aus der spätsargonischen Zeit sind bekanntermaßen spärlich überliefert, ganz im Gegensatz zu dem außerordentlich umfangreichen Urkundenmaterial dieser Epoche'. So läßt sich zwar aus den Königsinschriften von Sarkališrri, dem letyten großen Sargonidenherrscher (2217-2193) noch ein einigermaßen fundiertes Bild gewinnen ${ }^{2}$; über die in dieser Zeit wiedererstarkenden Städte des mesopotamischen Südens (und deren Ensis) jedoch kaum, da hier die historischen Quellen bisher fast ganz verschollen sind.

Dies gilt insbesondere für die Region Lagå̌-Girsu, die, nach dem Urkundenmaterial zu schließen, als wirtschaftlicher Knotenpunkt im sargonischen Reich große Bedeutung wiedergewonnen hatte ${ }^{3}$. Gerade hier sind wir mit der Tatsache konfrontiert, daß von einem so bedeutenden Ensi wie Lugal-ušmgal von Lagaš-Girsu ${ }^{4}$, der in

' Vyl. hierzu die von B. Foster gegebenen Daten in "Archives and Record-Keeping in Sargonic Mesopotamia" (ZA 72 [1982] 4-8), jewejls zum Stichwort NS (Narüm-Sîn) und SKS (Sarka)iăarri)). Fiir Angaben zum Fundort der einzelnen Archive ist A. Westenholz, Circulation of Goods 18, Anm. Iff. zu vergleichen.

2 Für die Inschriften Sarkališarrīs und seiner Nachfolger s. zuletzt I. Gelb/B. Kicnast, FAOS 7 (1990) 113-122 und 276-282.

3 S. B. Foster, Mesopotamia 9 (1982) 111, und J. J. Glassner, BBVO 5 (1987) 44.

4 Lugal-uðumgals Amtszeit als Ensi (zuvor war er Schreiber) begann in der späten Narām-Sîn-Zeit und endete vermutlich in der zweiten Hälfte der Regierungazeit des Königs Sarkaližarri; vgl. zuletzt Glassner, BBVO 5, 44. 
den zeitgenössischen Urkunden weit über 50 mal erwähnt wird ${ }^{5}$, keinerlei historische Inschriften auf uns gekommen sind".

Angesichts solcher Bedingungen ist für uns jede - noch so frag. mentarische oder stichwortartige - Information aus den Urkunden von Bedeutung, die historische Gegebenheiten jener Zeit reflektiert. Insbesondere dann, wenn sie vom direkten Aufeinandertreffen der Akkad-Könige mit dem mehrfach unterworfenen Süden berichtet.

Dies ist etwa der Fall bei der vermuteten Visite Narām-Sîns in Girsu ${ }^{7}$ und kann als gesichert gelten für die „Reise“ Sarkalisarris nach Kengir bzw. Nippur, dokumentiert in 2 Jahresdaten Sarkali-

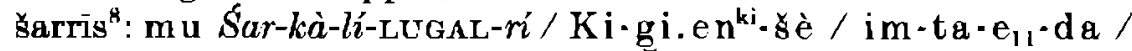
[2] sa g̃ g̃á (/ mu-ús-bi) ..Jahr, als Šarkališarrī zum ersten" Male nach Kengir herunterkam (folgendes Jahr) “. Weitere Hinweise auf die Anwesenheit von Surkališarri in Nippur bzw. Kengir geben uns eine Reihe von Wirtschaftsurkunden, die in direktem Zusammenhang mit seinem Aufenthalt im Süden stehen ${ }^{10}$. Aus diesen Urkunden, die im wesentlichen aus Umma ${ }^{11}$, Isin ${ }^{12}$ und Girsu ${ }^{13}$ stammen,

5S. Foster, ZA 72, 10 Anm, 27. Hervorzuheben ist hier vielleicht dus epigruphisch bemerkenswerte Prisma, das Lugal-usumgal in seiner früheren Tätigkeit als Schreiber hinterlassen hat (vgl. W. G. Schileijco, ZA 29 [1914/I5] 78-84).

A Dies hat wahrscheinlich als Fundzufall zu gelten. Nach dem Jahresdatum I'TT 2/ 2, 9, L 2955, ist cine Bautätigkeit von Lugal-ußumgal anzunehmen, die sicherlich in einer - nicht erhaltenen - Inschrift kommemoriert wurde.

; Vgl. Foster, „Notes on Sargonit Royal Progress“, JANES 12 (1980) 29 ff. Beachte jedoch Fosters eigene Reservatio zum Zusammenhang der verschiedenen, von ihm zur Reise Narăm-Sîns gesammelten Dokumente (JANES 12, 36). Wahrscheinlich sind insbesondere $K^{\top} T C 221-229$ einem underen historischen Kontext zuzuordnen, d.h. der Spätzeit Sarkališarris.

s Nur das Folge-Juhresdatum ist dokumentiert. Vgl. zum Text A. Westenholz. OSP II Nr. 100 viii 4-8' und OSP II 203. Zur altbab. Abschrift dieses Datums zulet Gelb/Kienust, FAOS 7, 58 sub D 35. Eine abweichende Interpretation bietet Foster, JANES 12, 39. Zu notieren ist die anagraphische(?) Schreibung Kigi.e $n^{k i}$ (anstelle von $\mathrm{Ki}-\mathrm{en}-\mathrm{g} \mathrm{i}^{\mathrm{k} 1}$ ) sowohl im sargonischen Original wie in der altbab. Abschrift.

"Bei der Ergänzung und Ühersetzung von [2] sag̃ - Tá durch „zum ersten Male folge ich Westenholz, ECTJ 23 ad (TMH) Nr. 27 i 2 , der diese Deutung aus einer Reihe von Parallelbelegen erschlossen hat. Die Verwendung des Zahlzeichens /2/ bleibt aber hier wie in den von Westenholz zitierten Texten merkwürdig.

10 CT 50, 52 iv 46-47: logal Ki-en-gi-šè i-g̃in-na-a „Als der König nach Kengir reiste"; MCS 9, 247: 29-30: lugal Ki-en-gi-šè ì-im-g̀in-na-a; BRM 3, 26: 4-5: lugal Nibru im-DL-a: BIN, 8 li34:8-9; $i$-nu ldYAs, / u-ur-da-ni.

11 CT 50, 54; MCS 9, 247; BRM 3, 26.

12 NBC 6848, ef. JANFA $12,37 \%$. und ZA 72, 18.

13 L 1212+L 4672 (JANES 12, 41 f.); L. 2940) (ITT 2/2; 8; JANES 12, 42). 
geht hervor, daß wohl der gesamte mesopotamische Süden anläßlich der Reise Šarkališarris nach Nippur seine Ergebenheitsadresse und natuirlich seine Abgaben zu entrichten hatte. Dies sowohl für den König und seine Familie als auch für die zahlreichen hohen akkadischen Funktionäre, die in den versehiedenen Städten Südmesopotamiens tätig waren und die zu diesem Anlaß beim König in Nippur versammelt wurden ${ }^{14}$. Neben diesen, hauptsächlich akkadischen Namens- und Funktionsträgern, haben aber vermutlich auch die Ensis, allen voran der bereits erwähnte Lugal-ušumgal von Lagaš-Girsu, Sarkališarrī ihre Aufwartung gemacht ${ }^{15}$.

Ein völlig anderes Bild für das Verhältnis von der Zentralmacht Akkad zu einer der Städte des Südens, Lagał-Girsu, ergibt sich aus einem zunächst eher peripheren spätsargonischen Brief, RTC 83.

In diesem Brief, der den Charakter einer Petition trägt, bemüht sich offenbar der Absender Puzur-Mama ${ }^{16}$, Ensi von Lagas, den Adressaten Sarkališarri(? $)^{17}$ zur Garantie von alten lagašitischen Territorialrechten zu bewegen:

Vs. $\quad$ l [lugal-mu $]^{?}$

$2\left[\grave{\mathrm{u}}-\mathrm{n} \mathrm{a}-\mathrm{du} \mathrm{u}_{11}\right]$

$3\left[{ }^{*} P\right] \dot{u}-{ }^{\lceil} z u r_{B}{ }^{d 1]}[M a-m a]$

4 [én]si-La[gasio $\left.{ }^{\mathrm{ki}}-\mathrm{ke}_{4}\right]$

5 'nal-bé-a

$6 \mathrm{l} \mathrm{Su}_{11}-\mathrm{lum}^{\mathrm{ki}}$

$71 \mathrm{E}_{2}{ }^{\text {Bis }}$ apin ${ }^{\mathrm{ki}}$
[Zu meinem 'Herrn'](?)

[sprich,]

das, was Puzur-Mama, der Ensi von Lagaš

sagt:

(Betrifft) Sulum (und)

Eapin.

14 Foster, JANES 12, 39, wollte in diesem Anlaß die Krönung Sarkališarris sehen. Diese These beruht auf einer problematisohen Ergänzung des Jahresdatums PBS 5, 38: 4, die nununehr durch die Veröffentlichung von OSP II 100 viii $4^{\prime}-8^{\prime}$ (beachte dic Raumverhältnisse vor sag̃-ğá!) widerlegt sein dürfte. Vgl. hierzu oben Anm. $y$ und Westenholz, OSP II 27 ad OSP II 100.

15 Viclleicht ist in diesem Zusammenhang BIN 8, 214: 9-10 zu sehen. Daß daneben in underem Zusammenhang dic Ensis von Lagas. Girsu nach $\Lambda$ kkade zu reisen hatten, zeigt z. B. ITT $2 / 2$, L 4690) iv 4 und I'TT 1, L 110417.

1A Ije Zeichenspuren von RTC $83: 3$, die das Autonym dee Ensi angeben, sind entgegen Sollberger. ArO 17, 30, nicht in [Lugal]-u \& [u mgal] zu orgänzen. Kopie und Kollation (durch A. Cavigneaux) ergeben, da $B$ die Zcichenreste in $*[P \mid \dot{u}-$

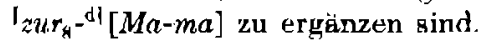

17 Diesc Ergänzung ergibt sich insbesondere aus den Zeilen 10-13 des Briefes, aus denen hervorgeht, dả der Adressat ein Nachfolgcr Naräm-Sîns gewegen sein muls. Hierfür knmmt am ehesten Sarkalisarri (kum einer seiner "Nachfolger ${ }^{*}$ ) in Betracht. Vgl. bereits Sollberger, AfO 17, 30. Besteht die Ergänzung zurecht. ergibt sich ein bisher nicht nachgewiesener Synohronismus Puzur-Mama - Sarkališarri. 
$8^{\lceil} \mathbf{u}_{4}{ }^{\top} S a r-m u-G \mathbf{r} \cdot[\mathbf{t}] \mathrm{a}^{(3)}$

9 ki-sur-ra-Laga $\mathbf{s}^{\prime k i l} \cdot[\mathrm{kam}]$

$10 \mathrm{Ur}{ }^{\mathrm{d}} \mathrm{Utu}-\mathrm{ke}{ }_{4}$

$11\left[{ }^{*} n\right] a m-e ́ n s i-\int U r i_{2}{ }^{k_{1} \mid}-m a$

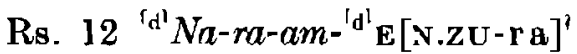

$13^{\top} \mathbf{i}-\mathbf{n} a-a k^{\top}-k a$

$142 \mathrm{ma}-\mathrm{na} \mathrm{kù} \cdot \mathrm{sig}_{17}$

$15 \mathrm{kadra} \mid \mathrm{b}-\mathrm{si}_{\mathrm{i}}-\mathrm{C}^{1}$

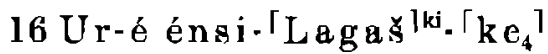

$17 \mathrm{ba}-\mathrm{da}-\mathrm{kar}$

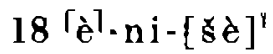

$19\left[\mathrm{x}^{\mathrm{q}} \mathrm{L} \mathrm{a}\right] \mathrm{ga}\left[\mathrm{s}^{\mathrm{ki}}-\quad\right]$ ca. 2 Zeilen abgebrochen

Rd. $22^{z}$ hé-eb-*MI- $\left.\right|_{X}$
Seit der Zeit Sargons (gehören sie)

zum Territorium von La-

gas.

Ur-Utu hat, als er das Amt des Ensi von $\mathrm{Ur}$

unter Narām-Sîn

ausgeübt hatte,

2 Minen Gold

als Geschenk dafür gemacht.

Ure, der Ensi von Lagaš, hatte (sie (ON) wieder) an sich genommen -

Was nun seine Reise anbetrifft:

.... Lagå ... .

$\cdots$

soll er...

Kommentar: Z. 1-2: Diese Frgänzung kann als sehr wahrscheinlich gelten, beruht sie doch auf einer großen Zahl von Parallelen innerhalb des Corpus der sargonischen Briefe. In lugal iat hier aicherlich der König (Sarkališrri) zu achen.

Z. 6: Zu $\mathrm{Su}_{11}-1 \mathrm{~m}^{\mathrm{k} .} \mathrm{vgl}$. RGTC 1, 147.

Z. 7: S. RGTC 1, 37. Dic in Z. 6 und 7 genannten Toponyme dürften wohl süd lich bzw. südwestlich von Girsu, im Grenzgebiet zu $\mathrm{U}_{r}$, zu lokalisieren sein.

Z. 16-17: In Anbetracht des Frgativa, realisiert in énsi-Lagaski-ke, muß kar als têerum, ekémum "wegnehmen" interpreticrt werden; vgl. schon A. Falkenstcin, AnOr. 30, 12 Anm. 6; anders F. Sollberger, AfO 17 (1954/56) 30, der mit "l'ensi de Lagå̆ s'enfuit“ eine nicht-ergativische Konstruktion unterstellt. Um dem Präfix /ba-/ gerecht au werden, übersetzc ich ba-da-kar reflexiv: „er hat an sich genommen".

Z. 18: Sollberger, AlO 17, 30, hatte die Zeichenreste [u]d-du-lí-[a-ta](?) gelesen und "Depuis des tempo frès anciens* übersetat. Nach einem Hinweis von D. O. Edzard hat Sollberger in [u]d-du - li - $[a-\ldots]$ wohl cine "Sandhi-Schreibung" für *u(d)-ul-lí-a gesehen. Eine solche Annahme scheint mir jedoch für einen sargonischen Brief kaum gegeben. - Für die Lesung und Ergänzung der Zeichenfolge

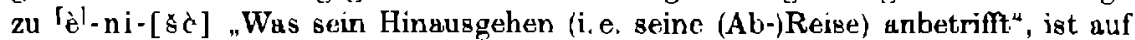
den sargonischen Brief A 636 7-11 (Z. Yang, Sargonic Inscriptions from Adab (1989) 123; vormals: Sollberger, JCS 10, $17 \mathrm{f}$ ) zu verweisen: è-ni-sè / Ki-babbar-

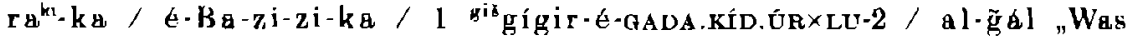
nun seine (Ab-)Reise anbetriff: In Kibabbara, im Hause des Bazizi, ist ein zweirädriger Wagen vorhunden". Unerklärt bleibt bei dieser Interpretation, warum in è-ni-§è jewcils cine "reine" hamfu-Form verwendet wird (also nicht è-a-nj- è). 
Eine formale, jedoch zeitlich und inhaltlich entfornte Parallele bietet è-ni-s c̀ g̈iri-zu mu-ži-DU.DU in TMH NF 3, 36 i 9 (urudu - kì) und CBS 10220+ (s. A. Sjöberg, JCS 34, 67) Vs. i 6'-7' é-d En-lill-lá-la $\dot{e}-n i / x k i-d u_{10}-g a$ àm ma-da-an-ku-k $u_{4}$. SehlieBlich jst zu noticren, daB in dem o.g. Jahresdatum dic verbale Wurzel $/ \mathrm{e}_{11} /:$ waradum, nicht wie hier $/ \mathrm{è} /:$ unasim benutzt wird.

Z. 22 Die Verbalform läßt sich vorläufig nicht mit Sicherheit ergänzen.

Der Brief vermittelt uns also eine Reihe singulärer Daten. Danach gehörten Sulum und Eapin seit der Zeit Sargons zum Gebiet von Lagax. Die bestehenden Territorialrechte wurden aber von Ur-Utu, dèm Ensi von Ur unter Narām-Sîn. korrumpiert: Er gab hierfür zwei Minen Gold „als Geschenk“ (an Narüm-Sîn?). In der Folge dieser Ereignisse gelang es dann aber Ure ${ }^{1 \mathrm{~s}}$, dem Ensi von Lagaš, die genannten Ortschaften wieder unter seine Kontrolle zu bringen. Möglicherweise erreichte er dies in der direkten Auseinandersetzung mit Ur ohne weitere Einbeziehung staatlicher akkadischer Institutionen ${ }^{19}$. Dennoch - oder vielleicht gerade wegen dieser prekären Umstände - wendet sich Puzur-Mama, der Ensi von Lagaš, in dieser Angelegenheit an den König.

Leider ist unser Brief in der Schlußpassage weitgehend zerstört, so daß über das definitive Ansinnen Puzur-Mamas Unklarheit besteht. Die Reste dieses Briefabschnittes ergeben jedoch immerhin, $\mathrm{da} B$ es sich um eine Bitte (hé-eb-MI- ${ }^{\prime} \mathbf{x}^{\prime}$ "er soll es ... im Zusammenhang mit dem Territorium von Lagaš (Z. 19) handelt. Aufgrund des Kontextes könnte man vermuten, $\operatorname{da} B$ das Briefende den Wunsch nach Territorialgarantie von Seiten des Königs enthielt. Ob diese im Rahmen einer Inspektionsreise oder Visite ( des Königs im Süden ${ }^{20}$ institutionell besiegelt werden sollte, bleibt leider spekulativ.

Wie auch immer die Petition des Puzur-Mama im einzelnen ausgesehen haben mag: Inhalt und Formulierung des Briefes sind nicht

IN Da der Ensi Ure m. W. nur in unserem Text bezeugt ist, bleibt seine chronologische Einordnung schwierig (zwischen Lugal-ušumgal und Puzur-Mama?). Vgl. hicrzu Fulkenstein, RLA 3, 387, der Ure in die Regierungszeit Naram-Sins daticren möchte, also vor Lugał-ušumgal.

19 Zum langwährenden Territorialstreit zwischen Lagas und Ur s. Falkenstein, AnOr. 30, 16 Anm. 6.

20 Von der wir in dicser Zeit aus keiner anderen Quelle wissen, es sei denn, RTC 221-229 seien doch mit F. Thureau-Dangin in die späte sargoniache Zeit oder postimperiale Epoche zu datieren und mil dieser Reise zu verbinden gegen Foster, der diese 'Texte in die späte Naràm-Sîn-Zeit datieren will und sie in Zusammenhang mit dem von ihm vermuteten Aufenthalt Naräm-Sins in Laga Girsu stellt. 
nur für das Corpus der sargonischen Briefe $\mathrm{e}^{21}$ ohne Parallele. Der Ensi von Lagaš fordert also über den König alte lagašitische Territorialrechte ein, vermutlich um den bisher unter den Sargoniden zugestandenen Einflußbereich von Lagaš-Girsu, und damit den des Puzur-Mama, wieder zu erweitern ${ }^{22}$. Eine derartige Konstellation ist zweifellos nur in der Spätzeit sargonidischer Herrschaft denkbar.

Diese Überlegungen lassen sich nun mit einiger Gewißheit durch die Selbstdarstellung des Ensi, späterhin Königs Puzur-Mama stützen.

Puzur-Mamas bedeutender Vorgänger im Amt des Ensi, Lugalušumgal $^{23}$, hat, soweit wir wissen, sowohl die späte Naram-Sîn-Zeit als auch einen wesentlichen 'Teil der Regierungszeit Sarkališarris erlebt. $\mathrm{Zu}$ welchem Zeitpunkt er starb und in Ure ${ }^{24}$ oder direkt Puzur-Mama seinen unmittelbaren Nachfolger fand, ist bisher unbekannt. Sicher scheint aber nunmehr über den Brief RTC 83, duß Puzur-Mama zum Ende der Regierungszeit des Sarkalisarri in Lagă̌-Girsu uls Ensi' ${ }^{2.3}$ fungierte und sich, vermutlich infolge der Wirren nach der Ermordung Surkalizarris ${ }^{26}$, den Titel lugal „König “ aneignete ${ }^{2 \bar{\gamma}}$, den vor ihm zwar eine Reihe altsumerischer Lagas-Herrscher beanspruchte ${ }^{28}$, nach $\mathrm{ihm}$ aber kein weiterer hoher Funktionär in Lagaš-Girsu mehr trug. In dieses Bild fügen sich die Aussagen der einzigen von Puzur-Mama erhaltenen historischen Inschrift (AO 11253) ${ }^{29}$, in der er sich „König von Lagaš‘ao nennt:

2 Eine Edition aller altsum. und sargonischen Briefe von B. Kienast und K. Volk wird in Kürze in FAOS erscheinen; vgl. hierzu Kienast, FAOS 7, 38 sub (8).

22 Der Hinweis auf die lagazitischen Torritorialverhältnisse unter Sargon (RTC 83:8) bedeutet wohl. Aaß Lagns sich scincrzeit in riner günstigen Ausgangsposition (anders als unter Rimus) befunden haben muB.

23 Vgl. Anm. 5; zu den Siegeln von Lugal-usumgal zuletzt. (kelb/Kienast, FAOS 7, 42 (S-13 und 14) und $46(S-32)$.

¿4 S. Anm. 18.

2s S. RTC 181 iv 1-2; vgl. zu diesem Text. Foster, Mes. 9, 40.

28 Vgl. J. Cooper, CRRA 26 (Deuth in Mesopotamia) (1980) $99 \mathrm{ff}$.

2: S. Falkenstein, RLA 3. 387; Sollberger, AfO 17, 31.

2s Vgl. hier die Zusammenstellung bei H. Behrens/H. Steible, FAOS 6, 224 f.

20 Veröffentlicht von $H$. de Genouillac, in: Revue de l'Histoirc des Religions 101 (19:30) $220 \mathrm{f}$.

31 AO 11253 ii 6'-8'; ein weiterer Beleg ist ITT 5, L 6758 R8. iv(?) 1'-3' (Bruchstiuck einer großen Tafel mit verschiedenen Summenformeln). 
ii $\left.1^{\prime}[\mathrm{mu} \cdot \mathrm{pà}-\mathrm{da}]\right]^{\text {? }}$

$2^{\prime}$ ['En-líl-lá $]^{?}-\mathrm{ke}_{4}$

$\left.3^{\prime}\right|^{\prime}{ }^{\prime}-$ sum-ma-

$4^{\prime d} \mathrm{Nin}-\tilde{\mathrm{g}} \mathrm{ir}-\mathrm{su}-\mathrm{ka}-\mathrm{ke} \mathrm{e}_{4}$

$5^{\prime}$ g̃éstú-sum-ma-

6' ${ }^{\mathrm{d}} \mathrm{En}-\mathrm{ki}-\mathrm{ka}-\mathrm{ke} \mathrm{t}_{4}$

$7^{\prime}$ ga-zi-kú-a-

$8^{\prime}$ d Nin-bur-sago-ka-ke

$9^{\prime} \mathrm{mu} \cdot \mathrm{d} \mathrm{u}_{10}-\mathrm{sa}_{4}-\mathrm{a}$ -

$10^{\prime}{ }^{d}$ Inanna-ka-ke

$1 \mathbf{l}^{\prime}$ [dumu]-tu-da-

$12^{\prime}\left[{ }^{\mathrm{d}} \mathrm{G}^{\mathrm{a}} \mathrm{a}\right]-\mathrm{du} \mathrm{u}_{10}$.tù m-[ga $]^{3}-\mathrm{ke}_{4}$ von Gatumdu;

$13^{\prime}$

(unbekannte Zeilenzahl abgebrochen)

iii $\mathbf{l}^{\prime} \Gamma_{\mathbf{X}}{ }^{l}[$

$2^{\prime}$ am $[a-t u]-d[a-n i]$

$3^{\prime}{ }^{d} \mathrm{Nin}-\mathrm{subur-kam}$

$4^{\prime}$ diğir-ra-ni

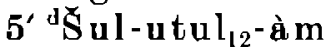

$6^{\prime} \mathrm{P}$ ù-zur $r_{8}-\mathrm{Ma}-\mathrm{ma}$

7 ' lugal-

$8^{\prime}$ Lagas $\left.{ }^{\mathrm{ki}} \cdot \mathrm{kam}\right]^{?}$

Rest abgebrochen
[(Puzur-Mama), der mit Namen berufen wurde von Enlil]; dem Stärke verliehen wurde von Ning̃irsu; dem Weisheit (ein-)gegeben wurde von Enki;

der mit 'rechter' Milch genährt wurde

von Ninhuursag̃a;

dem ein verheißungsvoller Name genannt wurde von Inanna;

der Sohn, geboren

]. . . .

seine leibliche Mutter

ist Ninšubura;

sein Familiengott

ist Sul-utul:

Puzur-Mama

[ist] ${ }^{?}$ der König

von Lagaš

Die gesamte Herrschertitulatur dieser Inschrift zeigt, daß sich Puzur-Mama an den ältesten lagašitischen Vorbildern orientiert ${ }^{31}$, vielleicht sogar bevorzugt an dem des Eannatum. Diese Rückorientierung gipfelt in der Adaption des Familiengottes ${ }^{32}$ Sul-utul ${ }^{33}$, der

31 Vgl. etwa Eannatum 2 i 7-8; ii 2-7 (FAOS 5/I 146); Eannatum 3 ii 1-2 (FAOS $5 /$ I $152 \mathrm{f}$ ).

32 G. J. Selz, mit dem ich das Problem einer gezielten Adaption eines Sippengottes diskutiert habe, hält es für mindestens ebenso wahrscheinlich, daß Puzur-Mama Sul-utul nicht adaptiert hat, sondern daß er der alten Patrizierfamilie des $\mathrm{Ur}_{\mathbf{r}}$ Nanse und seiner Nachfahren angehört hat und somit völlig legitim anf seinen Familiengott zurückgreifen konnte.

33 Die legendenhafte Lagaš-HerTscherliste BM 23103 Rs. iv 183-184 (s. Sollberger, JCS 21 [1967] 279-291) bezeichnet Zazaru als Familiengott deg Puzur-Mama, 
in seiner Funktion bereits unter Uru-inimgina durch Ninsubura abgelöst war. Beide Traditionen scheint Puzur-Mama indes auf seine Weise zu verknüpfen, indem er Ninsubura als seine "leibliche Mutter und Sul-utul als seinen Familiengott bezeichnet.

Zweifellos hat man sich bei der Beurteilung dieser Königsinschrift des Puzur-Mama das Problem der großen Überlieferungslücke historischer Texte zwischen Uru-inimgina und Puzur-Mama zu vergegenwärtigen. Dennoch scheint die Vermutung erlaubt, daß Puzur-Mama mit dieser Inschrift weniger in der Tradition seiner direkten Vorgänger ${ }^{34}$ steht, als daß er in gezieltem Rückgriff auf alte, ja älteste lagašitische Traditionen seinen Anspruch auf den Titel „König“ manifestiert. Diese Entwicklung dürfte sich in der späten Sarkališarri-Zeit angebahnt haben, als Puzur-Mama in Lagaš-Girsu noch Ensi war und den in seiner Thematik singulären Brief (RTC 83) verfaßte.

hier als ,Schreiber der Ninki“ tituliert. Vgl. hierzu Sollberger. JCS 21, 286 Anm. 73; Falkenstein, AnOr. 30, 114.

34 Dies sind nuch Uru-inimgina (énai, bzw. lugal-Laga $g^{k_{1}}$ ): Meszi, Ensi unter Sargon; Kituši (Rimus); Uru-inimgina (Sohn des Fngisa, Maniǒ́tūgu); Lugalułumgal (Naräm·Sîn/Sarkališarri), Ure (Sarkališarrī?). 